\title{
Boundary line analysis of the effect of water-filled pore space on nitrous oxide emission from cores of arable soil
}

R.M. LARK ${ }^{\mathrm{a}} \&$ A.E. MILNE ${ }^{\mathrm{b}}$

${ }^{\mathrm{a}}$ British Geological Survey, Keyworth, Nottinghamshire NG12 5GG, U.K. and ${ }^{\mathrm{b}}$ Rothamsted Research, Harpenden, Hertfordshire AL5 2JQ, U.K.

Correspondence: R.M. Lark. E-mail: mlark@bgs.ac.uk

Running title: Water-filled pore space and nitrous oxide emission

Key words: Greenhouse gases, anaerobicity, limiting factor, emission factor 


\section{Summary}

2 The boundary line has been proposed as a model of the effects of some variable on a

3 biological response, when this variable might limit the response in only some of a set

4 of observations. It is proposed that the upper boundary (in some circumstances the

5 lower boundary) represents the response function of interest. Boundary line analysis

6 is a method to estimate this response function from data. The approach has been

7 used to model the emission of $\mathrm{N}_{2} \mathrm{O}$ from soil in response to various soil properties.

8 However, the methods that have been used to identify the boundary are based on

9 somewhat ad hoc partitions of the data. A statistical model that we have presented

10 previously has not been applied to this problem in soil science, and we do so here to

11 represent how the water-filled pore space (WFPS) of the soil affects the rate of $\mathrm{N}_{2} \mathrm{O}$

12 emission. We derive a boundary line response that can be shown to be a better model

13 for the data than an unbounded alternative by statistical criteria. Furthermore, the

14 fitted boundary response model is consistent with past empirical observations and

15 modelling studies with respect to both the WFPS at which the potential emission

16 rate is largest and the measurement error for the emission rates themselves. We

17 show how the fitted model might be used to interpret data on soil volumetric water

18 content with respect to seasonal changes in potential emissions, and to compare

19 potential emissions between soil series that have contrasting physical properties.

- We obtain a boundary model of the effect of water-filled pore space on soil nitrous oxide emission

- The boundary model can be fitted by maximum likelihood allowing for measurement error.

- The boundary model indicates a maximum emission rate with water-filled pore space from $0.7-0.8$

- The model can be used to compare potential emission rates of soil with different properties 


\section{Introduction}

Nitrous oxide $\left(\mathrm{N}_{2} \mathrm{O}\right)$ is produced in soil by nitrification and denitrification. Microbial denitrification occurs when soil becomes anaerobic. Facultative anaerobic bacteria use nitrate as the electron acceptor in their respiration, reducing it to various forms dominated by $\mathrm{N}_{2} \mathrm{O}$ and $\mathrm{N}_{2}$. The proportions of these two products depend on factors such as soil redox potential and pH (Delwiche, 1981). Nitrous oxide is also produced as a by-product of nitrification, the oxidation of ammonium to nitrate. The relative importance of these two sources of $\mathrm{N}_{2} \mathrm{O}$ depends on local conditions (Stevens et al., 1997).

Nitrous oxide is an important greenhouse gas, and it has been estimated that a $\mathrm{CO}_{2}$ equivalent of $97 \mathrm{Tg} \mathrm{C}$ year $^{-1}$ is emitted from agricultural sources across continental Europe (Schulze et al., 2009). This, together with methane, is more or less balanced by the net sink for carbon provided by Europe's grassland and forest. With the intensification of agriculture and forestry a net flux of greenhouse gases to the atmosphere can be expected from agricultural and forest land of Europe (Schulze et al., 2009). We must be able to predict $\mathrm{N}_{2} \mathrm{O}$ emissions from soil under different conditions to formulate policy and to design interventions to mitigate this effect.

Various factors determine the rate of $\mathrm{N}_{2} \mathrm{O}$ emission from soil (Dobbie \& Smith, 2003). Soil organic carbon as a substrate for respiration is unlikely to be limiting on denitrification but the consumption of oxygen by aerobic microflora, stimulated by a supply of organic carbon, might promote the development of anaerobic conditions in which denitrification can occur (Groffman et al., 1987). Bacteria require a supply of nitrate and ammonium to sustain denitrification and nitrification, respectively, and the form in which nitrogen is available in soil affects the rate of $\mathrm{N}_{2} \mathrm{O}$ emission (e.g. Bayer et al., 2015). Both nitrification and denitrification respond to temperature (Smith et al., 1998). Soil pH has an effect on most microbially-mediated processes, and it influences the proportions of $\mathrm{N}_{2} \mathrm{O}$ and $\mathrm{N}_{2}$ in denitrification products (Delwiche, 1981). One of the most important factors that affects the rate of denitrification in soil is the development of anaerobic centres where the process can take place. This depends on factors that affect the rate of gaseous diffusion into soil such as compaction (Ball et al., 2000) and the proportion of the soil's pore space that is filled with water (water-filled pore space; WFPS; Smith et al., 1998). Because nitrification is an aerobic process, the relative contributions of the two processes to $\mathrm{N}_{2} \mathrm{O}$ emission also depends on the WFPS, see, for example, Bateman \& Baggs 
(2005).

These factors must be considered in any quantitative model for nitrous oxide emissions from soil. Some progress has been made towards process-based models including the DNDC (Li, 2000) and DAYCENT (Del Grosso et al., 2006) models.

Process modelling has also been used to investigate particular factors, such as the effect of WFPS. Rabot et al. (2015) used simulation modelling to investigate the effects of WFPS on gas transport and implications for denitrification and the emission of denitrification products from the soil. Their model showed a bell-shaped response to WFPS with the maximum emission rate at a WFPS in the interval [0.76, 0.79]. The WFPS that gave the maximum emission rate drifted from the bottom to the top of this interval with time during an experiment because of the increase in the $\mathrm{N}_{2} \mathrm{O}$ concentration gradient between the soil surface and the atmosphere. At smaller WFPS the rate of emission of $\mathrm{N}_{2} \mathrm{O}$ is limited by small denitrification rates because anaerobicity is reduced. At larger WFPS the rate of emission is limited by the rate of gaseous diffusion through the soil.

Process models give insight into the factors that contribute to $\mathrm{N}_{2} \mathrm{O}$ emissions from soil. Note, for example, how the modelling by Rabot et al. (2015) helps us to understand the factors that contribute to the non-linear effect of WFPS. However, process models may be challenging to use in practice because of the need for information on many soil properties, the propagation of error in model parameters and inputs and uncertainty about the model structure. Conen et al. (2000) considered that empirical models might be more useful in some circumstances, at least as submodels within broader process models.

Various empirical models have been used to predict $\mathrm{N}_{2} \mathrm{O}$ emission rates from agricultural soil. Conen et al. (2000) used a model based on soil mineral N content, WFPS and soil temperature, and the same variables were used in a similar approach by Smith \& Massheder (2014). Although these models are empirical they are not simple regressions. Rather they use the boundary line concept of Webb (1972). Webb (1972) proposed that, for many biological processes, the boundary (typically the upper boundary) in a scatter plot of the biological response (on the ordinate) against an environmental variable of interest (on the abscissa) expresses best the effect of the environmental variable. Specifically, it represents a maximum response given the value of the environmental variable, which will be expressed only if other factors are not limiting. The two empirical models cited above use boundary line 
responses to express the effects of WFPS and soil temperature on $\mathrm{N}_{2} \mathrm{O}$ emissions. The boundary line method was used by Schmidt et al. (2000) to examine the effects of temperature, soil nitrate content and WFPS. In earlier research the boundary line method was used to model the effects of soil properties specifically on denitrification rates (Elliot \& de Jong, 1993; Bergstrom \& Beauchamp, 1993).

Farquharson \& Baldock (2008) suggest that boundary line models might be particularly appropriate for modelling $\mathrm{N}_{2} \mathrm{O}$ emissions from soil because of the many factors which affect this process and cannot be controlled in an observational study, and the plausibility of the limiting factor interpretation of the boundary line. Nevertheless, they noted some limitations with the methods that had been used for the boundary line analysis (BLA). For example, Schmidt et al. (2000) obtained a boundary line model for the response of $\mathrm{N}_{2} \mathrm{O}$ emission rate to some factor by dividing the range of values of the factor into eight equal intervals, and then extracting the observation from within each interval that corresponded to the 99th percentile of $\mathrm{N}_{2} \mathrm{O}$ emission rates in that interval. A continuous function was then fitted to the resulting eight data points by ordinary least squares. This is a reasonable heuristic approach, and is similar to other methods published at the time of their study (Schnug et al., 1996) and since (Shatar \& McBratney, 2004). However, as Farquharson \& Baldock (2008) pointed out, these methods provide no statistical evidence that the boundary line is a plausible model of the particular data. Furthermore, they either disregard measurement error in the response variable or deal with it in an arbitrary way. Farquharson \& Baldock (2008) referred to previous research that we had undertaken with colleagues to develop exploratory methods to examine the plausibility of the boundary line interpretation of data (Milne et al., 2006a) and a statistical model for the boundary line which can be fitted by maximum likelihood (Milne et al., 2006b). The suggested that these methods be applied in studies on $\mathrm{N}_{2} \mathrm{O}$ production from soil stating that 'The adoption of BLA to define relationships could be of considerable benefit to model development as it provided a more appropriate way to define bivariate relationships where other factors cannot be controlled.' The papers by Milne et al. (2006a,b) demonstrated our BLA methods in examples from plant physiology, agronomy and studies on soil carbon. We are not aware of any studies that have applied our method to the study of $\mathrm{N}_{2} \mathrm{O}$ emission from soil. Therefore we decided to use it to investigate the effect of WFPS on $\mathrm{N}_{2} \mathrm{O}$ emission rate with data from a previous study on arable soil (Lark et al., 2004). 
The proposed methodology for BLA has two stages. In an initial exploratory analysis the evidence for an upper boundary line model, provided by a concentration of observations near the upper limit of the scatter plot, is examined by counting the number of upper vertices in the first few convex hull 'peels' (Eddy, 1982) of the scatter plot of the response variable against the environmental variable of interest. The convex hull of a set of data in a plane is the subset of points that are the vertices of the convex polygon which includes exactly all the data. The convex hull of a bivariate data set is its first peel. The convex hull of the remaining data after the first peel is removed is the second peel, and so on. In the exploratory analysis of data, these are compared with the expected number of vertices in the null case represented by a bivariate normal joint distribution of the two variables. We expect to see more vertices than are expected in the null case if the upper boundary of the scatter plot represents the limiting response to the variable on the abscissa of the plot. Milne et al. (2006a) describe the method. Second, the boundary line is then modelled as a function that censors a joint bivariate normal distribution of the underlying response variable, $y$, and the measured environmental factor, $x$, on the abscissa of the plot (Milne et al., 2006b). In summary, if the boundary line is described by $b(x)$, then a variate from the joint distribution $\{y, x\}$ where $y>\bar{y}=b(x)$ is replaced by $\{\bar{y}, x\}$. However, the response variable might be measured with error, and so observed variates $\{\breve{y}, x\}$ might occur above the boundary line. The model is fitted by finding maximum likelihood estimates of the bivariate normal distribution of $\{y, x\}$, parameters of the boundary function $b(x)$ and the variance of the measurement error, assumed to be a normal random variable with a mean of zero. By comparing the maximized likelihood for this distribution with the maximized likelihood of a bivariate normal joint distribution one may assess the weight of evidence for the boundary line model.

In this paper we use the methods of Milne et al. (2006a,b) to analyse a data set on rates of emission of $\mathrm{N}_{2} \mathrm{O}$ from cores of arable soil, and their WFPS. We used this variable so that we could compare the WFPS at the maximum rate of emission rate in the fitted boundary model with the results of the process modelling reported by Rabot et al. (2015). We use a somewhat different formulation of the censored model for the boundary line to that presented by Milne et al. (2006b). We used conditional densities, which allows a more straightforward treatment of measurement error for the response variable. This is presented in the next section, followed by an account 
of the data and specific analyses.

\section{Theory}

\section{The boundary line model}

The boundary line model is a bivariate distribution of an observed response variable, $\breve{y}$ and an independent covariate $x$. This model is based on a latent normal random variate $\mathbf{z}=\{y, x\}^{\mathrm{T}}$ with joint density function

$$
f(y, x)=\phi_{2}(\mathbf{z} \mid \boldsymbol{\mu}, \mathbf{C}),
$$

where $\phi_{2}()$ denotes the bivariate normal density function for a random variate with mean vector $\boldsymbol{\mu}$ and covariance matrix $\mathbf{C}$. The variate, $\mathbf{z}$, is censored by a boundary function $b(x \mid \boldsymbol{\beta})$ with parameters in $\boldsymbol{\beta}$, to give a censored variate $\overline{\mathbf{z}}=\{\bar{y}, x\}^{\mathrm{T}}$. In the case of an upper boundary:

$$
\overline{\mathbf{z}}=\{\min (y, b(x \mid \boldsymbol{\beta})), x\}^{\mathrm{T}} .
$$

We assume that the independent variable is known without error, as in the general linear model, and that the observed response variable, $\breve{y}$, arises from the observation of $\bar{y}$ with a normal error of mean zero and standard deviation $\sigma_{\mathrm{e}}$, such that the distribution of the observed value conditional on $\bar{y}$ is:

$$
\breve{y} \mid \bar{y} \sim \mathcal{N}\left(\bar{y}, \sigma_{\mathrm{e}}\right) .
$$

The boundary line model has three sets of parameters. These are the parameters of the censoring function, in $\boldsymbol{\beta}$, the parameters (means and covariances) of the latent bivariate normal random variate, and the observation error $\sigma_{\mathrm{e}}$. Our objective is to estimate these parameters by maximum likelihood, given the observed values $\left\{\breve{y}_{1}, \breve{y}_{2}, . . \breve{y}_{n}\right\}$ and $\left\{x_{1}, x_{2}, . . x_{n}\right\}$. To obtain the appropriate likelihood function we require the joint density for $\breve{y}$ and $x$ conditional on the parameters:

$$
f\left(\breve{y}, x \mid \boldsymbol{\beta}, \boldsymbol{\mu}, \mathbf{C}, \sigma_{\mathrm{e}}\right) .
$$

34 For brevity we drop the parameters from the density functions. Following familiar properties of conditional densities we may write

$$
f(\breve{y}, x)=f(\breve{y} \mid x) f(x),
$$



$194 \quad y:$

$$
\mu_{y \mid x}=\mu_{y}+\left(x-\mu_{x}\right) \frac{\operatorname{Cov}\{x, y\}}{\sigma_{x}^{2}},
$$

195

$$
\sigma_{y \mid x}=\sigma_{y} \sqrt{1-\rho^{2}},
$$

$$
\gamma_{\mathrm{U}}=\frac{\beta \sqrt{2 \pi}}{2 \pi \sigma_{y \mid x} \sigma_{\mathrm{e}}\left\{1-\Phi\left(\frac{\mu_{y \mid x}-b(x)}{\sigma_{y \mid x}}\right)\right\}}
$$


205

206

$$
\eta_{\mathrm{U}}=1-\Phi\left(\frac{\breve{y}-b(x)-\alpha}{\beta}\right)
$$

$$
\zeta_{\mathrm{U}}=\Phi\left(\frac{b(x)}{\sigma_{y \mid x}}\right)
$$

$$
\alpha=\frac{\sigma_{\mathrm{e}}^{2}\left(\bar{y}-\mu_{y \mid x}\right)}{\sigma_{y \mid x}^{2}+\sigma_{\mathrm{e}}^{2}},
$$

207 and

$$
\beta^{2}=\frac{\sigma_{y \mid x}^{2} \sigma_{\mathrm{e}}^{2}}{\sigma_{y \mid x}^{2}+\sigma_{\mathrm{e}}^{2}} .
$$

208

209

210

211

$$
\gamma_{\mathrm{L}}=\frac{\beta \sqrt{2 \pi}}{2 \pi \sigma_{y \mid x} \sigma_{\mathrm{e}} \Phi\left(\frac{\mu_{y \mid x}-b(x)}{\sigma_{y \mid x}}\right)},
$$

$$
\eta_{\mathrm{L}}=\Phi\left(\frac{\breve{y}-b(x)-\alpha}{\beta}\right)
$$

212 and

$$
\zeta_{\mathrm{L}}=1-\Phi\left(\frac{b(x)}{\sigma_{y \mid x}}\right) .
$$

213

For some proposed set of parameters $\boldsymbol{\beta}, \boldsymbol{\mu}, \mathbf{C}$ and $\sigma_{\mathrm{e}}$, and a pair of observed 214 values $\breve{y}$ and $x$ one may compute the density from Equation (11). Treating each of a 215 set of $n$ observations as independent, one may compute the negative log-likelihood 216 for a set of parameter values, given the observations, as

$$
\ell=-\sum_{i=1}^{n} \log f\left(\breve{y}_{i} \mid x_{i}\right) .
$$

217 Materials and methods

218 Data collection

219 Our data are drawn from a study on the spatial variation of $\mathrm{N}_{2} \mathrm{O}$ emissions from 220 soil cores from a regular transect (Lark et al. 2004). The measurements were made 221 on incubated intact cores so that temperature was fixed, but other factors (such 
as water content) varied between soil cores to reflect variation in the field. Ideally, measurements would be made in situ in the field. However, spatial analysis and BLA require large data sets, and it is difficult to collect these in the field without confounding spatial with temporal variation. We chose, therefore, to make measurements on incubated intact cores, given previous experience of making useful measurements of denitrification and mineralization this way (Ryden et al., 1987; Webster \& Goulding, 1989; Hatch et al., 1990).

A full account of the data collection is given by Lark et al. (2004), but we provide an outline here. Soil samples were taken within a period of seven hours in the autumn of 2000 on a straight transect, with a spacing of $4 \mathrm{~m}$ and to give 256 sample points across the farm of the former Silsoe Research Institute in Bedfordshire in Eastern England. All fields traversed by the transect had been under a cereal crop in the summer of 2000, and had been either recently drilled with an autumnsown crop or were under stubble. At each site a gouge auger of length $150 \mathrm{~mm}$ and diameter $44 \mathrm{~mm}$ was pushed fully into the soil, twisted and removed. Four cores were taken in this way at each site. The cores were transported with a minimum of delay to a cold room at $4^{\circ} \mathrm{C}$, and were kept at this temperature until they were analysed.

In subsequent laboratory analysis one core was selected from each site, and its fresh weight and length were recorded. Cores were placed in a 1-litre Kilner jar and pre-incubated at $15^{\circ} \mathrm{C}$ for $17-24$ hours with the jar lids in position to prevent desiccation of the core, but unsealed to allow some gas transfer from the jar. After pre-incubation the jars were flushed with laboratory air and re-sealed with a rubber gasket and clamped in position so that they were gas-tight. An initial sample (20 $\mathrm{ml}$ ) of the gas headspace was collected and injected into an evacuated vaco tube. The jars were incubated at $15^{\circ} \mathrm{C}$ for 24 hours, and then two further 20 -ml samples from the headspace were collected. Within a few days the gas samples were injected into an Ai93 gas chromatograph which analysed them for $\mathrm{N}_{2} \mathrm{O}$ by an electron capture detector (ECD). The rate of emission was determined from the change in concentrations of $\mathrm{N}_{2} \mathrm{O}$ in the headspace of the incubation jar.

After incubation the core was cut in half and the moisture content of one half of the core was determined by oven-drying to constant weight at $105^{\circ} \mathrm{C}$ allowing the determination of volumetric water content. The dry bulk density was determined. The rate of $\mathrm{N}_{2} \mathrm{O}$ emission could then then expressed on an area basis as in previously- 
cited modelling studies (here $\mathrm{g} \mathrm{N} \mathrm{ha}{ }^{-1}$ day $^{-1}$ ). Other analyses were undertaken on the soil, including soil organic carbon content (SOC) by a combustion method following Tabatabai \& Bremner (1991).

Ideally the saturated water content of the soil would be determined directly by saturating the core and then determining its volumetric water content. This value for the saturated water content would then be used to compute WFPS of the field-moist soil. However, this was not required in the original study and further destructive analyses were done on the cores so that material was not available to do this measurement subsequently. For this reason we chose to compute the total pore space of the soil $\left(\mathrm{cm}^{3} \mathrm{~cm}^{-3}\right)$ from the measured bulk density on the assumption of a particle density of $2.65 \mathrm{~g} \mathrm{~cm}^{-3}$ (Hall et al., 1977), as proposed by Minasny et al. (1999) when no soil physical data other than bulk density are available. We recognize that this introduces an approximation into our data on WFPS because soil particle density may vary, and, furthermore, the total porosity might differ from saturated water content. Our soil samples were from arable sites only (excluding waste ground and field boundaries), therefore the variation in SOC was small (see Table 1) and so this approximation seems reasonable. The approach has been used to determine WFPS for modelling microbial processes in soil in a range of studies (e.g. Wu et al., 2015; Franzluebbers, 1989; Linn \& Doran, 1984). The WFPS was computed from this total porosity and the measured volumetric water content of the field-moist soil.

It is useful to note two findings from a later study in which soil cores were taken from a longer transect over more heterogeneous land uses (Haskard et al., 2010). All protocols were identical, the exception was that the head space samples from the incubation jars were placed in vials on a Perkin Elmer Turbo Matrix 110 Headspace autosampler (Perkin Elmer, Waltham, MA). The autosampler was linked to a Perkin Elmer Clarus 500 gas chromatogram (GC) (Perkin Elmer) by a fused silica transfer line to allow the automatic analysis of samples. The samples in this latter study were allocated at random to batches for measurement of rates of $\mathrm{N}_{2} \mathrm{O}$ emission. Analysis of these data showed that the effect of batch (and so the length of storage time of the sample) was negligible and statistically insignificant (Haskard et al., 2010). Duplicate measurements were made on 78 cores from this latter study, which have been analysed subsequently to estimate the measurement error standard deviation. The estimated standard deviation of measurement error was $0.46 \log \mathrm{g}$ 
$\mathrm{N}$ ha ${ }^{-1}$ day $^{-1}$.

The objective of the present study is to examine the effect of WFPS on rates of $\mathrm{N}_{2} \mathrm{O}$ emission from arable soil. The original sampling transect was regular, so included 10 cores from tracks, ditches and waste ground under rough vegetation.

Data from these cores were not used in the analysis reported here. We also excluded the first two cores from the headland of the northernmost field on the transect. This field was on the lightest soil formed over the Lower Greensand, and both cores were markedly compacted by the auger.

\section{Data analysis}

Exploratory analysis. In the boundary line statistical model observations are assumed to be drawn from a bivariate normal variable with an upper censor on the values of the response variable that depends on the value of the environmental variable. To make these assumptions plausible transformation of the variables may be necessary. The data on rates of $\mathrm{N}_{2} \mathrm{O}$ emission were transformed to natural logarithms for analysis given their markedly skewed distribution. The data on WFPS were not markedly skewed, but, as proportions, they cannot be regarded as normally distributed and so were transformed to logits (natural logarithms) before analysis. The response variable in our boundary line analysis, variable $\breve{y}$ in Equation (11), was $\log \mathrm{N}_{2} \mathrm{O}$ emission rate, and the independent variable, $x$, in Equation (11), was the logit of WFPS.

In circumstances where a boundary line model is appropriate, and the sampling is sufficiently wide-ranging to cover a range of conditions with different limiting factors, one would expect to find a concentration of observations in a scatter plot of $\breve{y}$ against $x$ near the boundary. This might be evident when the plot is examined, but we do not want to rely on visual assessment. It would be difficult to compare consistently the density of observations near a putative boundary and the concentration that would be expected under an unbounded model. Therefore, we prefer to use an objective statistical test of the density of observations near the boundary of interest. This was first described by Milne et al. (2006a). In this test the number of upper vertices in the first few convex hull peels of the scatter plot of the transformed flux and WFPS data are counted and compared with expected numbers under the null hypothesis of a bivariate normal distribution. The first convex hull peel of a scatter plot corresponds to the observations that are on the convex polygon that 
exactly encloses all the data points. The points on the convex hull are vertices of this polygon. If one removes the points on the convex hull of the first data set (these are called the first peel of the data) a convex hull can be determined for the remaining points. The observations that are on the vertices of this second convex hull constitute the second peel of the data.

Consider a peel of the scatter plot of our response variable $(y)$ on the ordinate against the potentially limiting variable $(x)$ on the abscissa. We number the vertices from 1 to $N$ clockwise on the convex hull where the first vertex is $\mathbf{v}_{1}=\left\{x_{1}, y_{1}\right\}$ such that if

$$
x_{1}=\min \left\{x_{i}\right\}_{i \in\{1, \ldots, N\}} \quad \text { and if } \quad\left(x_{1}=x_{j}, j \in\{2, \ldots, N\}\right) \quad \text { then } y_{1} \leq y_{j} .
$$

The upper convex hull is defined as the ordered set of vertices $\mathbf{v}_{1}, \mathbf{v}_{2}, \ldots \mathbf{v}_{k}$ where

$$
x_{k}=\max \left\{x_{i}\right\}_{i \in\{1, \ldots, N\}} .
$$

If an upper boundary is a plausible model of the relation between $\breve{y}$ and $x$ then we would expect to find more vertices in the first few peels of the scatter plot than are expected under the bivariate normal null model. We followed Milne et al. (2006a) by counting the number of vertices of the upper convex hull in the first 5 to 10 peels of the data. Hueter (1994) showed that the asymptotic distribution of the number of vertices in the first peel of a bivariate normal random variate is normal. Milne et al., (2006a) conjectured that the numbers of vertices in successive peels can be approximated as a normal random variable; Monte Carlo simulations supported this. They used the output from these simulations to find emulators for the number of vertices in successive peels of bivariate normal variates, and their variances. In the procedure that we used here the number of vertices in the first 5 peels was compared to the number expected under a null hypothesis of bivariate normality, and this was repeated for the first $6,7 \ldots 10$ peels. This was done because a concentration of vertices is expected where the peel is close to any boundary function, but this might not be true of early peels of the data in the presence of measurement error. Because these six hypotheses constituted a multiple hypothesis test, and the hypotheses are not independent (the number of vertices in the first $n+1$ peels is the number in the first $n$ plus the number in the $n+1$ th), we applied the false discovery rate control procedure for hypothesis testing with non-independent hypotheses proposed by Benjamini \& Yekeutieli (2001). Details of this procedure are given by Milne et al. (2006a). 
Boundary line model. Schmidt et al. (2000) used a bell-shaped function for the boundary response of $\mathrm{N}_{2} \mathrm{O}$ emission to WFPS:

$$
\mathcal{F}_{\mathrm{N}_{2} \mathrm{O}}(\vartheta)=\varphi_{\max } \exp \left\{\frac{-2(\vartheta-0.72)^{2}}{0.074}\right\},
$$

where the WFPS (a dimensionless proportion) is denoted by $\vartheta$ and $\mathcal{F}_{\mathrm{N}_{2} \mathrm{O}}(\vartheta)$ denotes the boundary $\mathrm{N}_{2} \mathrm{O}$ flux at this value of WFPS with a maximum value of $\varphi_{\max }$ when $\vartheta=0.72$. Note that this equation is rescaled from Schmidt et al. (2000) who specified WFPS as a percentage, and that they define this function for $0.3<\vartheta<0.93$. Rabot et al. (2015) used the same function. In this study, we selected an equivalent expression for the boundary function on the log scale and with WFPS on the logit scale:

$$
\log \left\{\mathcal{F}_{\mathrm{N}_{2} \mathrm{O}}(\vartheta)\right\}=\beta_{0}-\beta_{2}\left(\operatorname{logit}(\vartheta)-\beta_{1}\right)^{2}
$$

In this formulation $\beta_{0}$ is the logarithm of $\varphi_{\max }$; the maximum flux occurs when $\operatorname{logit}(\vartheta)=\beta_{1}$ and $\beta_{2}$ is a scaling parameter, which is zero if the boundary is a constant (not dependent on WFPS). Other functions have been used to model this effect, including a quadratic (Wu et al., 2015), and could be used to model the boundary function.

We fitted this model as a boundary line to our data by finding values of the parameters $\beta_{0}, \beta_{1}$, and $\beta_{2}$ that maximized the likelihood computed with Equation (20) where the function in Equation (24) is substituted for the general boundary function $b(x)$ in Equation (11). This was done on the R-platform for statistical computing ( $\mathrm{R}$ Core Team, 2014) with the optim procedure and the quasi-Newton BFGS algorithm for optimization (Broyden, 1970). When the set of parameters that maximized the likelihood in Equation (20) was found, we evaluated the Hessian matrix of the likelihood with respect to the parameters and obtained from it a covariance matrix for the estimation error of the parameters (see Dobson, 1990). We recognize that the likelihood computed with Equation (20) treats the observations as independent, which requires independent random sampling. Our data were not collected this way, but we make the assumption as a first approximation, and use the independent estimate of the standard deviation of the measurement error (referred to above) to check for evidence of bias in the variance parameters of the model that could arise from the lack of independence.

One way to evaluate the boundary line model is to compare its fit with a simpler alternative in which the two variables considered are modelled as a bivari- 
ate normal random variate (Milne et al., 2006b). A multivariate normal model was fitted to the observations by minimizing the negative log-likelihood. The minimized negative log likelihood, $\ell$, for the boundary model can be compared with that for the multivariate normal model. However, the latter model has five parameters (the means for the two variables, their variances and their correlation). The boundary model has the same parameters (for the underlying bivariate normal process) in addition to three parameters of the censoring boundary line and the standard deviation of observation error. These extra parameters mean that the boundary line model must fit at least as well as the multivariate normal, as judged by the value of $\ell$, and might be expected to fit better, even in a case where the multivariate normal model holds. It is necessary, therefore to account for the additional parameters in the boundary line model when making the comparison. This comparison can be made with Akaike's information criterion (AIC) (Akaike, 1973). The AIC is computed by

$$
\mathrm{AIC}=2 \ell+2 \mathcal{P},
$$

where $\mathcal{P}$ is the number of parameters in the model. The second term in Equation (25) is a penalty for model complexity. In any comparison the model with the smallest AIC is selected. Although the AIC is not a formal significance test, selection of the model with the smallest AIC minimizes the expected information loss through the selection decision (Verbeke \& Molenberghs, 2000).

\section{Results}

Exploratory statistics are given in Table 1 . Note that the number of vertices in the upper convex hulls of the first 5 to 10 peels all exceed the numbers expected under a null hypothesis of multivariate normality. All six of these null hypotheses can be rejected with the false discovery rate controlled at 0.05, i.e. the expected proportion of rejected null hypotheses that are actually true is no larger than 0.05. Summary plots are shown in Figure 1.

Table 2 gives the results from the fitting of the boundary line. The negative log-likelihood is markedly smaller for the boundary line model than for the alternative multivariate normal model. There are four more parameters in the boundary line model, but the AIC is still substantially smaller for the boundary line model indicating that it is to be preferred to the multivariate normal alternative. This is consistent with the results for the vertices in the convex hull peels of the data. The 
fitted boundary model is shown in Figure 2 together with a $95 \%$ confidence interval for the boundary line obtained from the covariance matrix of the boundary function parameters assuming the estimation errors are normal.

The parameter $\beta_{2}$ of the boundary model is positive for physically plausible cases (i.e. with the boundary convex upward) and zero if the boundary line is flat. The estimate of this parameter for the data analysed in this paper, given in Table 2 , is 0.54 with a $95 \%$ confidence interval [0.11,0.97], which is consistent with a physically plausible non-constant boundary line function.

The parameter $\beta_{1}$ of the fitted model, 1.19, (Table 2) is the logit of the WFPS at which the maximum boundary emission rate occurs. On back-transformation this is equivalent to a WFPS of 0.77 . The $95 \%$ confidence interval for the backtransformed parameter, assuming a normal estimation error, is $[0.69,0.83]$. This is consistent with the process model results reported by Rabot et al. (2015) who found maximum fluxes at WFPS values between 0.757 and 0.798 ; the variation was attributed to temporal effects during the period when the soil is wetted and variation in soil bulk density. It is also comparable with the boundary line model reported by Schmidt et al. (2000) for which the maximum flux was at WFPS $=0.72$.

Figure 2 shows the fitted boundary line model on the scatter plot of the transformed rate of $\mathrm{N}_{2} \mathrm{O}$ emission against WFPS. This shows the maximum value where logit WFPS is 1.19. Also shown, as two dotted lines, is the $95 \%$ confidence interval for the boundary line obtained by sampling from the estimated distribution of the three parameters $\beta_{0}, \beta_{1}$ and $\beta_{2}$.

The estimated standard deviation of the measurement error, given in Table 2 , is $0.53 \log \mathrm{g} \mathrm{N} \mathrm{ha}^{-1}$ day $^{-1}$ with a $95 \%$ confidence interval $[0.35,0.71]$. This is consistent with the standard deviation of duplicate measurements from the second study (Haskard et al., 2010) reported above $\left(0.46 \log \mathrm{g} \mathrm{N} \mathrm{ha}^{-1}\right.$ day $\left.^{-1}\right)$. Figure 3 shows the profile likelihood for this parameter (the maximized value of the likelihood with this particular parameter fixed at different values). The profile likelihood is smooth with a minimum near to the estimate.

\section{Case Study}

In this section we give two examples to demonstrate the insight and information that the BLA model can provide, using the model with parameter estimates given in Table 2. In the first example we use data from sensors that measure volumetric 
water content (VWC) of the soil (5TE sensors, Decagon Devices, Pullman, WA). A cluster of 12 sensors was installed at a depth of $10 \mathrm{~cm}$ as part of a larger sensor network on a grassland site at Hollin Hill in Yorkshire, Northern England. Although this was a grassland site and our BLA model was estimated for arable soil, the data are used here for illustration. Two measurements of soil bulk density were made from soil removed when the cluster of sensors was installed, and these were used to estimate total porosity assuming a mineral particle density of $2.65 \mathrm{~g} \mathrm{~cm}^{-3}$ (Hall et al., 1977). We computed the mean VWC for all sensors in the cluster for each day from 1st January 2013 to the end of July of the same year. We then scaled the mean VWC to mean WFPS given the estimate of total porosity. Figure 4(a) shows these values. The horizontal line on the graph is at WFPS $=0.77$, the value at which the boundary line model for $\mathrm{N}_{2} \mathrm{O}$ emissions is largest. We call the term

$$
\mathcal{W}=\exp \left\{-\beta_{2}\left(\operatorname{logit}(\vartheta)-\beta_{1}\right)^{2}\right\},
$$

the WFPS factor, a dimensionless quantity which is 1 when the WFPS, denoted by $\vartheta$, allows maximum $\mathrm{N}_{2} \mathrm{O}$ flux and less than 1 otherwise. This is plotted for each day in Figure 4(b). Solid symbols indicate that the soil is wetter than the optimum for $\mathrm{N}_{2} \mathrm{O}$ flux, and open symbols where it is drier. Note the initial increase in the factor, which is caused by drying of the soil. Within the first 50 days there were some heavy rain events, which increased WPFS above 0.77. This caused marked transient reductions in the WPFS factor, but much of the time it was close to 1 . From about day 80 there was an overall decline in the WFPS factor because of drying of the soil with a few episodic increases in the factor that resulted from heavy rain. This analysis illustrates how potential $\mathrm{N}_{2} \mathrm{O}$ emissions from soil vary temporally. During the winter months illustrated here, the WFPS factor was mostly close to 1 , with episodic reductions because of inadequate air-filled pore space to allow gaseous diffusion out of the soil. Applied fertilizer $\mathrm{N}$ during this period is more likely to be lost to denitrification than during the spring when the WFPS factor shows a downward trend as the soil becomes too dry for the development of anaerobic centres (open symbols in Figure 4(b)). This provides a basis for the interpretation of sensor data for improved nitrogen management, or to develop generalized regional guidance on timing of applications or refinement of emission factors to account for regional weather patterns and soil conditions.

In our second example we used the BLA model, presented in Table 2, to examine the variation between potential rates of $\mathrm{N}_{2} \mathrm{O}$ emission in contrasting soil 
series. We assumed in all cases that the soil was at field capacity (assumed to be a tension of $5 \mathrm{kPa}$ ) and we used values of porosity and VWC at field capacity reported in soil survey memoirs. For the Cuckney series, a coarse loamy sand (Jarvis et al., 1984) the WFPS is 0.38 at field capacity. The corresponding value of the WFPS factor, computed with Equation (26), is 0.2. We can infer, therefore, that the soil is too-well aerated at field capacity because of its coarse texture for the widespread development of anaerobic centres where denitrification can take place. In contrast the Formby series, a loamy medium sand (Fordham, 1986) has a WPFS of 0.75 at field capacity. The corresponding value of the WFPS factor is 0.99 ; the soil is close to the optimum for $\mathrm{N}_{2} \mathrm{O}$ emission with respect to water content, wet enough for the development of anaerobic centres, but not so wet that it would inhibit diffusion of $\mathrm{N}_{2} \mathrm{O}$ out of the soil. The Ragdale series, a stagnogley in chalky clay drift (Burton, 1986) has a WFPS of 0.86 at field capacity, the WFPS factor is 0.80. Because the WFPS is larger than 0.77 , we can infer that the emission of $\mathrm{N}_{2} \mathrm{O}$ is somewhat limited by the slow rate of gaseous diffusion from the soil. These calculations give insight into how physical differences between soil types affect their potential for $\mathrm{N}_{2} \mathrm{O}$ emission when they are all at a standard water potential. Although the WFPS factor itself is not an emission factor for the various soil series (other potential limiting factors may apply), it could be used to rescale standard IPCC emission factors for soil to reflect variation between soils with different hydraulic properties that result from differences in texture inherited from contrasting parent materials.

\section{Discussion}

Our analysis shows that the boundary line is a plausible model for our data. The convex hull test provided evidence to reject the null hypothesis that the data were from a bivariate normal distribution, and the AIC for the boundary line model was smaller than that for the bivariate normal model. As noted in the previous section, the parameters for the fitted model were consistent with those reported elsewhere for the WFPS at which the maximum rate of emission occurs, and with an estimate of measurement error for our laboratory protocol. This consistency with previous empirical results and results from process modelling is encouraging and indicates that the BLA concept and our methods are plausible ways to model this particular response of the soil system.

Our analysis provides empirical support for the process model developed by 
Rabot et al. (2015). It could also be used for the development of empirical models such as those of Conen et al. (2000) and Smith \& Massheder (2014) which use the BLA concept. The calculation of $\mathrm{N}_{2} \mathrm{O}$ emissions in the current methodology of the Intergovernmental Panel on Climate Change (IPCC) does not take account of variation in climate or soil, which limits its usefulness because, for example, interventions to reduce the application of fertilizer nitrogen to soil with a WFPS close to the optimum for $\mathrm{N}_{2} \mathrm{O}$ emission would not affect inventory calculations. The use of process models to improve this is limited by their requirement for soil information. The BLA methodology presented here to estimate parameters of models in the style of Conen et al. (2000) could provide a basis for modelling in the IPCC framework because it takes better account of soil variation and our understanding of its effects. The case studies presented in the previous section illustrate how the BLA models could be used to explore how soil with contrasting physical properties might differ with respect to the likely rate of $\mathrm{N}_{2} \mathrm{O}$ emission, and how to interpret temporal data on soil moisture content with respect to the likely effect on the rate of $\mathrm{N}_{2} \mathrm{O}$ emission. This could provide a basis for refined advice on fertilizer use and timing, and improved emission rates for IPCC inventory.

Our boundary line model describes the limiting effect of a single explanatory variable, but more than one variable might be potentially limiting on a soil response. For example, temperature and $\mathrm{pH}$ might limit the rate of $\mathrm{N}_{2} \mathrm{O}$ emission from soil. In a situation where we consider more than one limiting factor, the response of the soil system might be described by von Liebig's law of the minimum (von Liebig, 1863)

$$
y=\min \left\{f_{1}\left(x_{1}\right), f_{2}\left(x_{2}\right), \ldots, f_{n}\left(x_{n}\right)\right\},
$$

where $y$ is the response variable and $x_{i}$ are the independent variables that limit $y$ according to the functions $f_{i}$ where $i=1, \ldots, n$. Under the law of the minimum one of these factors is limiting in any one case, and the $j$ th factor is limiting in some case if

$$
y=\min \left\{f_{1}\left(x_{1}\right), f_{2}\left(x_{2}\right), \ldots, f_{n}\left(x_{n}\right)\right\}=f_{j}\left(x_{j}\right) .
$$

This hypothesis could be tested for a suite of candidate limiting factors to determine which is limiting for each observation of the response variable. This would require an appropriate inferential method to compare the limiting factors for any observation in terms of the distance of the observation from each respective boundary line. This is a topic for further work. 
One way to develop the law of the minimum is to model interactions between different variables we observe that determine possible limiting effects on the soil response. For example, the WFPS and soil organic carbon content might interact to determine the 'anaerobiosis' limiting factor, whereas the nitrate and soil organic carbon might interact to determine a 'substrate' limiting factor. In this case the law of the minimum might be written as

$$
y=\min \left\{f_{\text {anaerobiosis }}\left(x_{1}, x_{2}\right), f_{\text {substrate }}\left(x_{3}, x_{4}\right), \ldots\right\}
$$

In this latter case, the boundary-line model must be extended to three or more dimensions with a boundary plane described by each function within the braces on the right-hand side of Equation (29).

In the log-likelihood function for the BLA model given in Equation (20) the observations are treated as independent. This assumption requires independent random sampling to be fully justified, which was not the case in our example, and will not be true for many studies where data are obtained on regular grids or transects. The extension of the boundary line model to the situation with spatial dependence could be based on a linear model of coregionalization (Journel \& Huijbregts, 1978) for the latent normal variate $\{y, x\}$, which underlies the boundary model. However, the derivation of the likelihood function for the model parameters under this joint distribution remains a challenge for further research.

\section{Conclusions}

The BLA approach with the censored normal model is an attractive method to estimate the limiting effects of soil factors on rates of $\mathrm{N}_{2} \mathrm{O}$ emission, and the BLA model that we have fitted and presented is consistent with previous modelling, and experimental results. Our BLA method has a theoretical basis that enables us to test the evidence for a boundary response, and to quantify the uncertainty of model parameters with confidence intervals. These give us an insight into the precision of the parameter estimates and so can be used to assess the uncertainty in any predictions of rates of $\mathrm{N}_{2} \mathrm{O}$ emission.

We have shown how the WFPS factor, derived from the BLA model and with parameters estimated from data, can be used to interpret real-time data on soil water content, and to indicate whether and how this variable can be expected to limit rates of $\mathrm{N}_{2} \mathrm{O}$ emission at particular times. We have also shown the possibility of 
577 using the model to scale the emission factors for $\mathrm{N}_{2} \mathrm{O}$ from contrasting types of soil,

578 which offers a way to improve the greenhouse gas inventory in the IPCC framework.

579 Further work is needed on the BLA methodology to account for spatial dependence

580 and to fit more general models for potential limiting effects of several factors.

\section{Acknowledgements}

582 This paper is published with the permission of the Director of the British Geological

583 Survey (NERC). We are grateful to Dr Barry Rawlins for the sensor data from Hollin

584 Hill. We have no conflicts of interest to declare. 


\section{References}

Akaike, H. 1973. Information theory and an extension of the maximum likelihood principle. In: Second International Symposium on Information Theory (ed. B.N. Petov \& F. Csaki), pp. 267-281. Akademia Kiado, Budapest.

Ball, B.C., Horgan, G.W. \& Parker, J.P. 2000. Short-range spatial variation of nitrous oxide fluxes in relation to compaction and straw residues. European Journal of Soil Science, 51, 607-616.

Bateman, E.J. \& Baggs, E.M. 2005. Contributions of nitrification and denitrification to $\mathrm{N} 2 \mathrm{O}$ emissions from soils at different water-filled pore space. Biology and Fertility of Soils, 41, 379-388.

Bayer, C., Gomes, J., Zanatta, J.A., Costa, F., Viera, B., de Cassia Piccolo, M., Dieckow, J. \& Six, J. 2015. Soil nitrous oxide emissions as affected by longterm tillage, cropping systems and nitrogen fertilization in Southern Brazil. Soil and Tillage Research, 146, 213-222.

Benjamini, Y. \& Yekutieli, D. 2001. The control of the false discovery rate in multiple testing under dependency. Annals of Statistics, 29, 1165-1188.

Bergstrom, D.W. \& Beauchamp, E.G. 1993. Relationship between denitrification rate and determinant soil properties under barley. Canadian Journal of Soil Science, 73, 567-578.

Broyden, C. G. 1970. The convergence of a class of double-rank minimization algorithms. Journal of the Institute of Mathematics and its Applications, 6, 76-90.

Burton, R.G.O. 1986. Soils in Cambridgeshire III, Sheet TL54 (Linton), Soil Survey Record No. 94, Soil Survey of England and Wales, Harpenden.

Conen, F., Dobbie, K.E. \& Smith, K.A. 2000. Predicting N2O emissions from agricultural land through related soil parameters. Global Change Biology, 6, $417-426$.

Delwiche, C.C. 1981. The nitrogen cycle and nitrous oxide. In: Denitrification, Nitrification and Atmospheric Nitrous Oxide (ed. C.C. Delwiche), pp 1-15, John Wiley \& Sons, New York. 
Del Grosso, S.J., Parton, W.J., Mosier, A.R., Walsh, M.K., Ojima, D.S., \& Thorton, P.E. 2006. DAYCENT National-Scale Simulations of Nitrous Oxide Emissions from Cropped Soils in the United States. Journal of Environmental Quality, 35, 1451-1460.

Dobbie, K. E. \& Smith, K. A. (2003), Nitrous oxide emission factors for agricultural soils in Great Britain: the impact of soil water-filled pore space and other controlling variables. Global Change Biology, 9, 204-218.

Dobson, A.J. 1990. An Introduction to Generalized Linear Models, Second Edition Chapman and Hall, London.

Eddy, W.F. 1982. Convex hull peeling. In: Compstat 1982, Part 1 Proceedings in Computational Statistics (eds H. Caussinus, P. Ettinger, R. Tomassone), pp. 42-47. Physica Verlag, Heidelberg.

Elliot, J.A. \& de Jong, E. 1993. Prediction of field denitrification rates: A boundaryline approach. Soil Science Society of America Journal, 57, 82-87.

Farquharson, R. \& Baldock, J. 2008. Concepts in modelling $\mathrm{N}_{2} \mathrm{O}$ from land use. Plant and Soil, 309, 147-167.

Fordham, S.J. 1986. Soils in Surrey I, Sheet TQ05 (Woking), Soil Survey Record No. 90, Soil Survey of England and Wales, Harpenden.

Franzluebbers, A.J. 1999. Microbial activity in response to water-filled pore space of variably eroded southern Piedmont soils. Applied Soil Ecology, 11, 91-101.

Groffman, P.M., Tiedje, J.M., Robertson, G.P. \& Christensen, S. 1988. Denitrification at different temporal and geographical scales: proximal and distal controls. In: Advances in Nitrogen Cycling in Agricultural Ecosystems (ed. J.R. Wilson), pp. 174-192. Commonwealth Agricultural Bureaux, Perth, Australia.

Hall, D.G.M., Reeve, M.J., Thomasson, A.J. \& Wright, V.F. 1977. Water retention, porosity and density of field soils. Soil Survey Technical Monograph No. 9. Soil Survey of England and Wales, Harpenden.

Haskard, K.A., Welham, S.J. \& Lark, R.M. 2010. A linear mixed model with spectral tempering of the variance parameters for nitrous oxide emission rates from soil across an agricultural landscape. Geoderma, 159, 358-370. 
Hatch, D.J., Jarvis, S.C. \& Philipps, L. 1990. Field measurement of nitrogen mineralization using soil core incubation and acetylene inhibition of nitrification. Plant and Soil, 124, 97-107.

Hueter, I. 1994 The convex hull of a normal sample. Advances in Applied Probability, 26, 855-875.

Jarvis M.G., Allen, R.H., Fordham, S.J., Hazelden, J., Moffat, A.J. \& Sturdy, R.G. 1984. Soils and their Use in South East England. Soil Survey of England and Wales Bulletin No 15. Lawes Agricultural Trust (Soil Survey of England and Wales), Harpenden.

Journel, A.G. \& Huijbregts, Ch.J. 1978. Mining Geostatistics. Academic Press, San Diego CA.

Lark, R.M., Milne, A.E., Addiscott, T.M., Goulding, K.W.T, Webster, C.P. \& O'Flaherty, S. 2004. Scale- and location-dependent correlation of nitrous oxide emissions with soil properties: an analysis using wavelets. European Journal of Soil Science, 55, 611-627.

Li, C. 2000. Modeling trace gas emissions from agricultural ecosystems. Nutrient Cycling in Agroecosystems, 58, 259-276.

Linn, D.M., \& Doran, J.W. 1984. Effect of water-filled pore space on carbon dioxide and nitrous oxide production in tilled and nontilled soils. Soil Science Society of America Journal, 48, 1267-1272.

von Liebig J. 1863. The Natural Laws of Husbandry. Walton and Maberly, London.

Milne, A.E., Wheeler, H.C. \& Lark, R.M. 2006a. On testing biological data for the presence of a boundary. Annals of Applied Biology 149, 213-222.

Milne, A.E., Ferguson, R.B. \& Lark, R.M. 2006b. Estimating a boundary line model for a biological response by maximum likelihood. Annals of Applied Biology 149, 223-234.

Minasny, B., McBratney, A.B. \& Bristow, K.L. 1999. Comparison of different approaches to the development of pedotransfer functions for water-retention curves. Geoderma, 93, 225-253. 
R Core Team 2014. R: A language and environment for statistical computing. R Foundation for Statistical Computing, Vienna, Austria. http://www.R-project. org/

Rabot, E., Cousin, I. \& Hénault, C. 2015. A modeling approach of the relationship between nitrous oxide fluxes from soils and the water-filled pore space. Biogeochemistry, 122, 395-408.

Ryden, J.C., Skinner, J.H. \& Nixon, D.J. 1987. Soil core incubation system for the field measurement of denitrification using acetylene inhibition. Soil Biology $\&$ Biochemistry, 19, 753-757.

Schmidt, U., Thöni, H. \& Kaupenjohann, M. 2000. Using a boundary line approach to analyze $\mathrm{N}_{2} \mathrm{O}$ flux data from agricultural soils. Nutrient Cycling in Agroecosystems, 57, 119-129.

Schnug E., Heym J. \& Murphy D.P. 1996. Establishing critical values for soil and plant analysis by means of the Boundary Line Development System (BOLIDES). Communications in Soil Science and Plant Analysis, 27, 2739-2748

Shatar T.M. \& McBratney A.B. 2004. Boundary-line analysis of field-scale yield response to soil properties, Journal of Agricultural Science, 142, 1-7.

Schulze, E.D., Luyssaert, S. , Ciais, P., Freibauer, A., Janssens, I.A. et al. 2009. Importance of methane and nitrous oxide for Europe's terrestrial greenhousegas balance. Nature Geoscience, 2, 842-850.

Smith, K.A. \& Massheder, J. 2014. Predicting nitrous oxide emissions from Nfertilized grassland soils in the UK from three soil variables, using the B-LINE 2 model. Nutrient Cycling in Agroecosystems, 98, 309-326.

Smith, K.A., Thomson, P.E., Clayton, H., McTaggart, I.P. \& Conen, F. 1998. Effects of temperature, water content and nitrogen fertilisation on emissions of nitrous oxide by soils. Atmospheric Environment, 32, 3301-3309.

Stevens, R.J., Laughlin, R.J., Burns, L.C., Arah, J.R.M. \& Hood, R.C. 1997. Measuring the contributions of nitrification and denitrification to the flux of nitrous oxide from soil. Soil Biology \& Biochemistry, 29, 139-151. 
Tabatabai, M.A. \& Bremner, J.M. 1991. Automated instruments for determinations of total carbon, nitrogen, and sulfur in soils by combustion techniques. In: Soil analysis: modern instrumental techniques, 2nd Edition (ed. K.A. Smith), pp. 261-286. Marcel Dekker, New York.

Turban, S. 2010. Convolution of a truncated normal and a centred normal variable. http://www. columbia.edu/ st2511/notes/Convolution $\% 20$ of $\% 20$ truncated $\%$ 20normal\%20and\%20normal.pdf Accessed 25th November 2015.

Verbeke, G. \& Molenberghs, G. 2000. Linear Mixed Models for Longitudinal Data. Springer, New York.

Webb R.A. 1972. Use of the boundary line in analysis of biological data. Journal of Horticultural Science, 47, 309-319

Webster, C.P. \& Goulding, K.W.T. 1989. Influence of soil carbon content on denitrification from fallow land during autumn. Journal of the Science of Food and Agriculture, 49, 131-142.

Wu, L., Rees, R.M., Tarsitano, D., Zhang, X., Jones, S.K. \& Whitmore, A.P. 2015. Simulation of nitrous oxide emissions at field scale using the SPACSYS model. Science of the Total Environment, 530-531, 76-86. 
Table 1. Exploratory statistics.

\begin{tabular}{|c|c|c|c|c|}
\hline & $\begin{array}{r}\mathrm{N}_{2} \mathrm{O} \text { emission } \\
\text { rate/ } \\
\mathrm{g} \mathrm{N} \mathrm{ha}^{-1} \text { day }^{-1} \\
\end{array}$ & $\begin{array}{r}\log \mathrm{N}_{2} \mathrm{O} \\
\text { emission rate/ } \\
\log \mathrm{g} \mathrm{N} \mathrm{ha}^{-1} \text { day }^{-1}\end{array}$ & $\begin{array}{r}\text { Water-filled } \\
\text { pore space/ } \\
\text { logit }\left(\mathrm{cm}^{3} \mathrm{~cm}^{-3}\right)\end{array}$ & $\begin{array}{r}\text { Soil organic } \\
\text { carbon } \\
\% \text { by mass }\end{array}$ \\
\hline Mean & 65.8 & 3.56 & 0.74 & 2.40 \\
\hline Median & 43.0 & 3.76 & 0.69 & 2.46 \\
\hline Quartile 1 & 15.0 & 2.71 & 0.42 & 2.09 \\
\hline Quartile 3 & 89.5 & 4.49 & 1.04 & 2.75 \\
\hline Minimum & 0.5 & -0.69 & -0.33 & 1.21 \\
\hline Maximum & 333.0 & 5.81 & 2.46 & 4.68 \\
\hline $\mathrm{SD}$ & 67.8 & 1.29 & 0.47 & 0.50 \\
\hline skewness & 1.6 & -0.66 & 0.61 & -0.05 \\
\hline
\end{tabular}

Number of upper vertices in successive convex hull peels

\begin{tabular}{lrrr}
\hline Hulls & $\begin{array}{r}\text { Expected number } \\
\text { of upper vertices }\end{array}$ & Observed number & $P$-value* \\
\hline & & & \\
$1-5$ & 37 & 44 & $3.0 \mathrm{E}-3$ \\
$1-6$ & 45 & 56 & $8.0 \mathrm{E}-5$ \\
$1-7$ & 53 & 63 & $1.0 \mathrm{E}-3$ \\
$1-8$ & 62 & 71 & $2.5 \mathrm{E}-3$ \\
$1-9$ & 70 & 80 & $2.0 \mathrm{E}-3$ \\
$1-10$ & 78 & 87 & $6.7 \mathrm{E}-3$ \\
\hline
\end{tabular}

* Null hypothesis: vertices arise from a bivariate normal process. With false discovery rate control at 0.05 all null hypotheses are rejected. 
Table 2. Boundary line fitting results. The parameters $\beta_{0}, \beta_{1}$ and $\beta_{2}$ are parameters of the boundary line model given in Equation (24), and $\sigma_{\mathrm{e}}$ is the standard deviation of the measurement error, given in Equation (3). The negative log residual-likelihood and number of parameters in each model are $\ell$ and $\mathcal{P}$ respectively. The AIC is defined in Equation (25).

\begin{tabular}{lrrr}
\hline Boundary model & & & \\
Parameter & Estimate & Standard error & $\begin{array}{r}95 \% \text { confidence } \\
\text { interval }\end{array}$ \\
\hline & & & \\
$\beta_{0}$ & 4.99 & 0.24 & {$[4.52,5.46]$} \\
$\beta_{1}$ & 1.19 & 0.21 & {$[0.79,1.61]$} \\
$\beta_{2}$ & 0.54 & 0.22 & {$[0.11,0.97]$} \\
& & & \\
$\sigma_{\mathrm{e}}$ & 0.53 & 0.09 & {$[0.35,0.71]$} \\
\hline \hline
\end{tabular}

Comparison with multivariate normal model

\begin{tabular}{lcc} 
& Boundary model & Multivariate normal \\
\hline$\ell$ & 560.3 & 580.2 \\
$\mathcal{P}$ & 9 & 5 \\
AIC & 1138.7 & 1170.5 \\
\hline
\end{tabular}




\section{Figure Captions}

1. Summary plots of (transformed) rates of emission and water-filled pore space. Scatter plots of $\log$ rate of $\mathrm{N}_{2} \mathrm{O}$ emission against (a) WFPS on the original scale and (b) WFPS on the logit scale. Histograms of (c) $\log$ rate of $\mathrm{N}_{2} \mathrm{O}$ emission and (d) logit of WFPS

2. Fitted boundary model for (transformed) rates of emission and water-filled pore space. The dotted line shows the $95 \%$ confidence interval for the boundary line

3. Profile likelihood for standard deviation of measurement error. Values $\hat{\sigma}_{\mathrm{e}}$ and $\hat{\sigma}_{\mathrm{d}}$ are, respectively, the maximum likelihood estimate and the estimated between-duplicate standard deviation of data collected in the study of Haskard et al. (2010). The vertical dotted line shows the $95 \%$ confidence interval of $\hat{\sigma}_{\mathrm{d}}$

4. (a) Daily mean water-filled pore space for a cluster of 12 sensors at Hollin Hill, N. Yorkshire, from 1st January 2013 to late July in the same year. The horizontal line is at 0.77 at which the WFPS factor, see Equation (26), is largest. (b) WFPS factor, see Equation (26), plotted with open symbols where the water- filled pore space is less than 0.77 and closed symbols where it is larger or equal to 0.77 . 
(a)

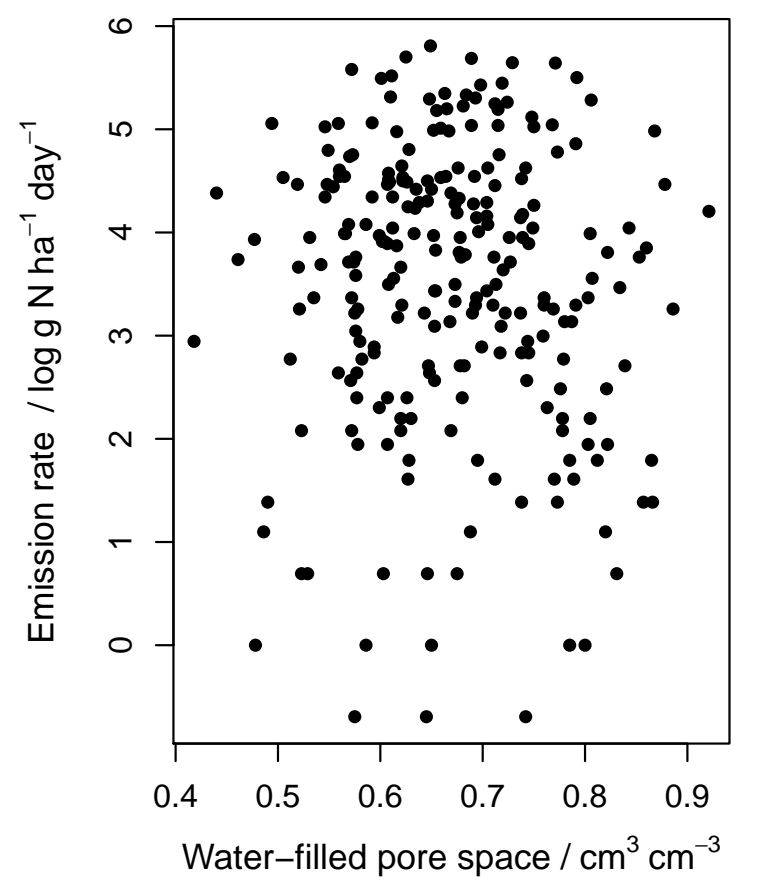

(c)

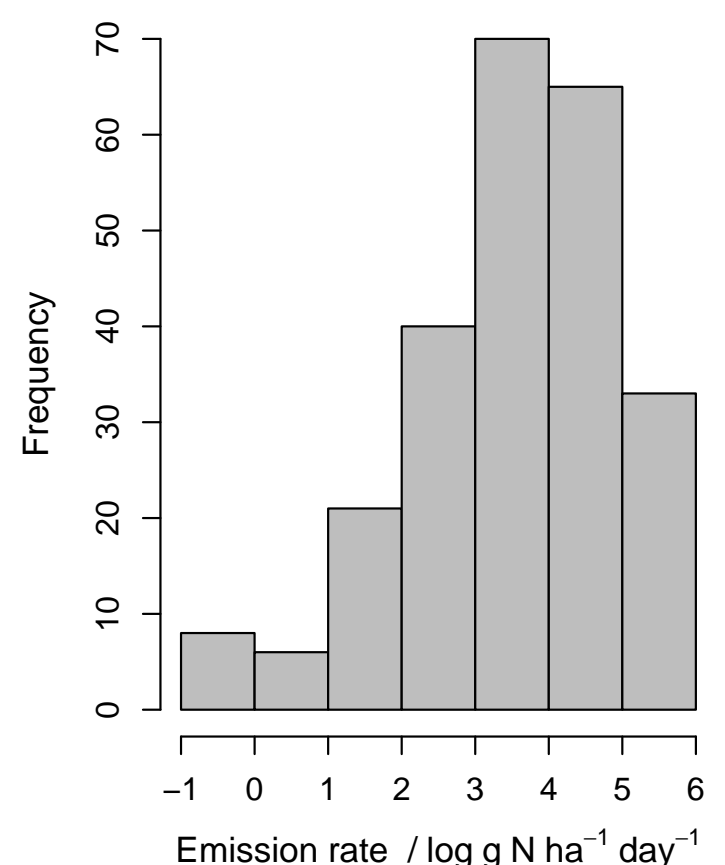

(b)

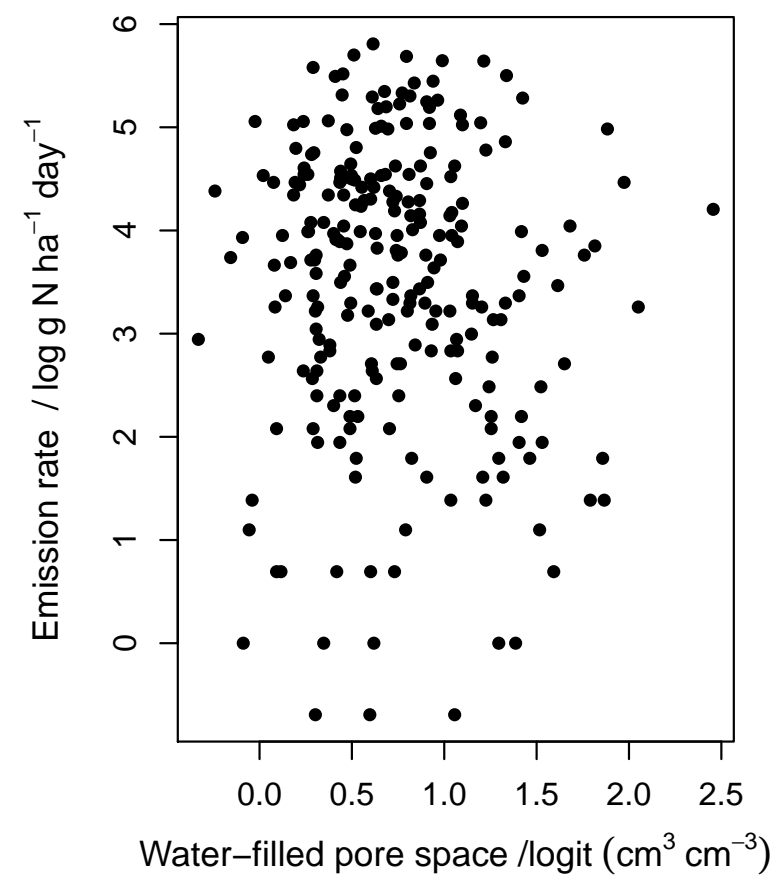

(d)

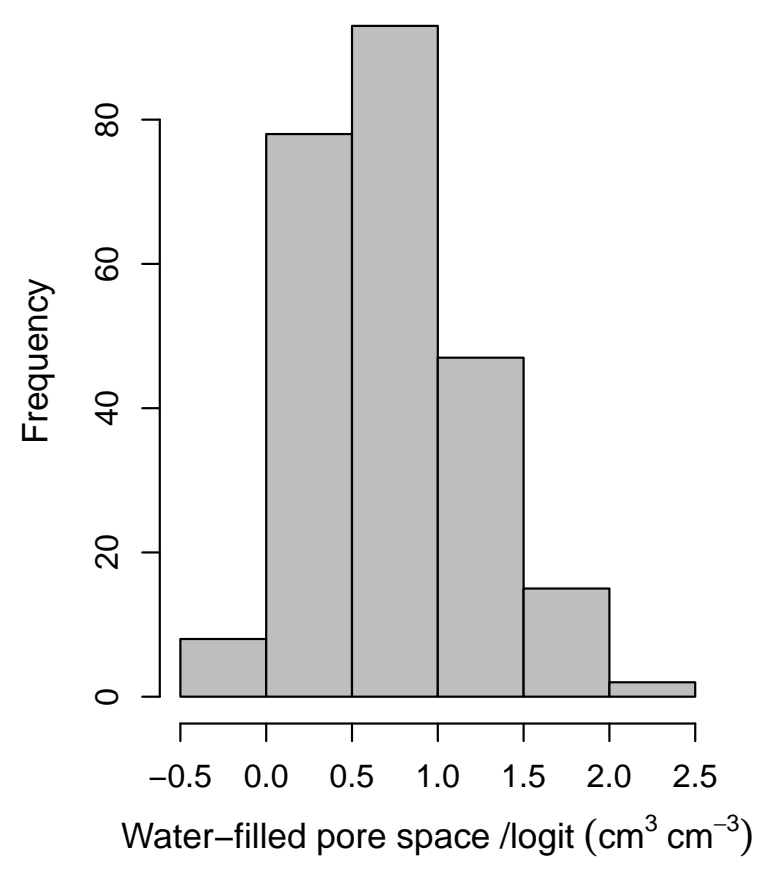

Figure 1: 


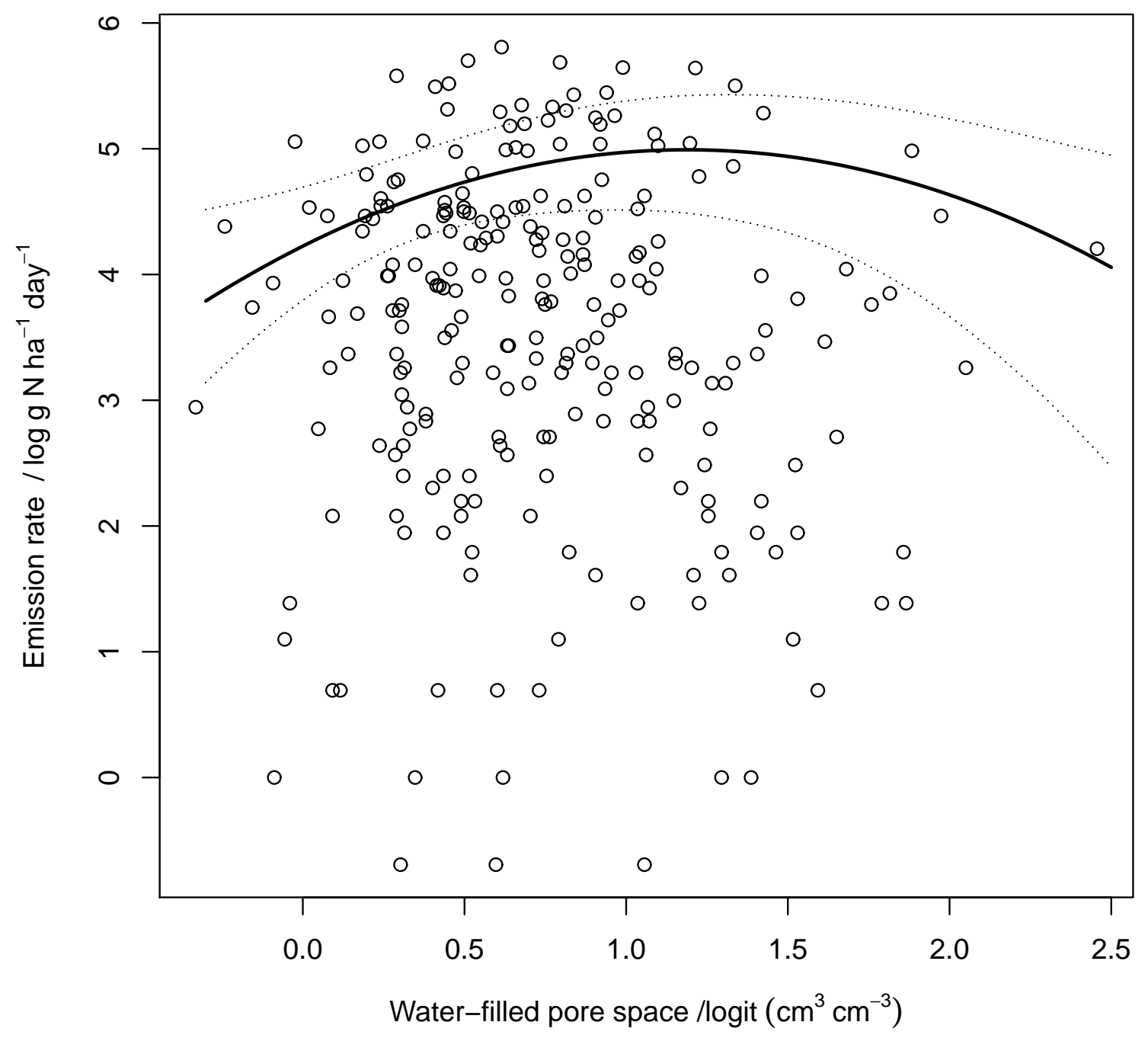

Figure 2: 


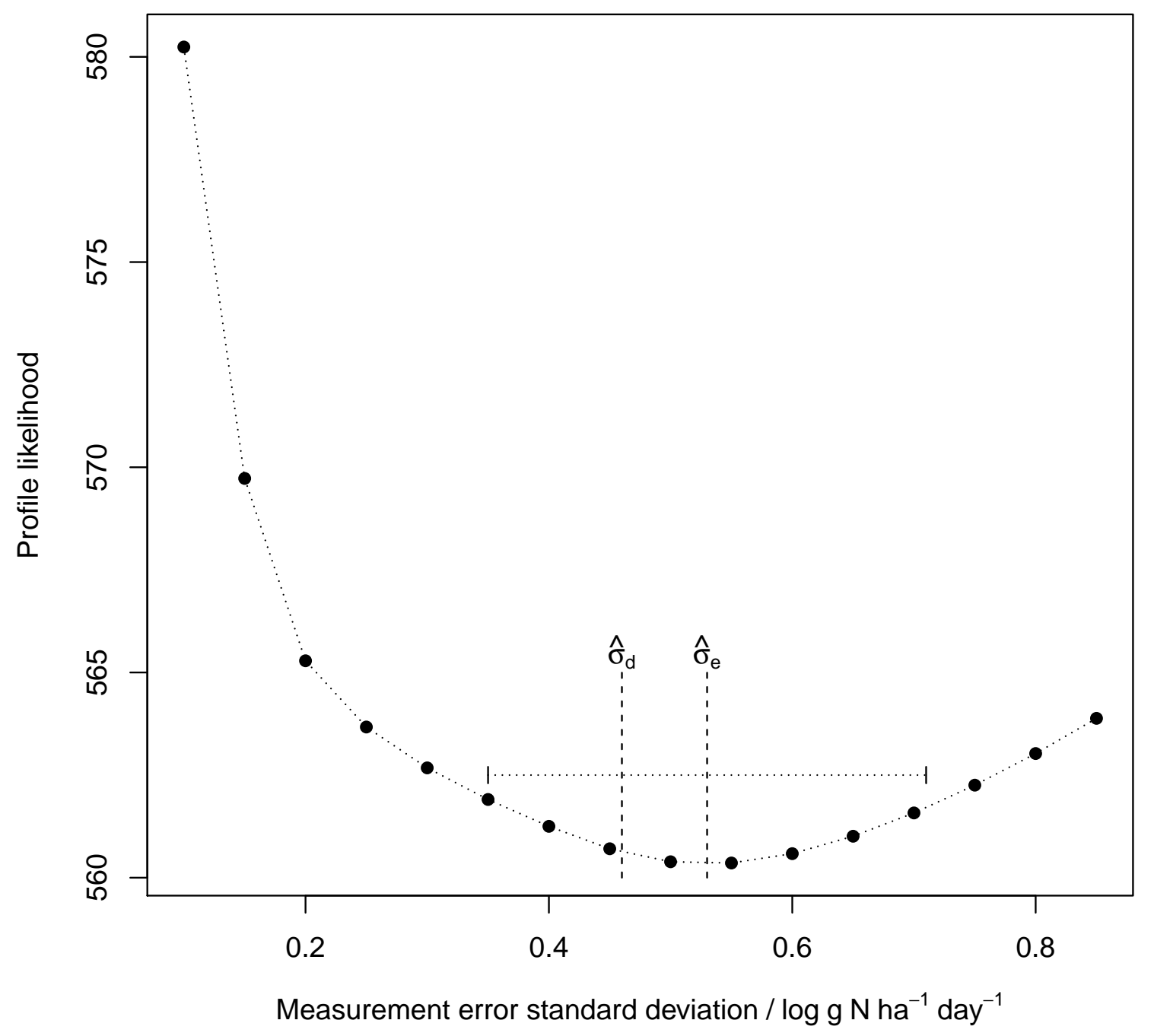

Figure 3: 
(a)

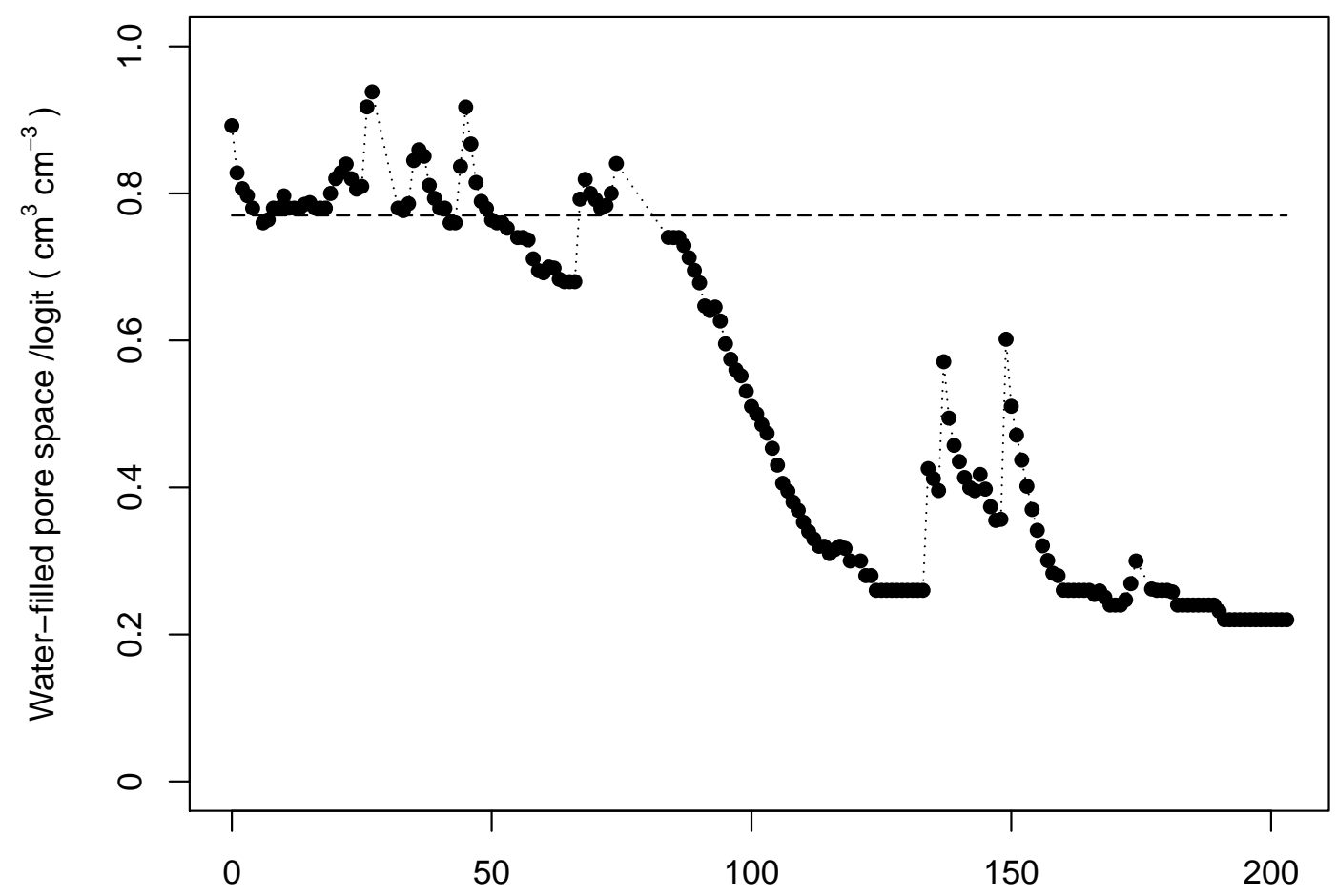

Days post 1st January 2013

(b)

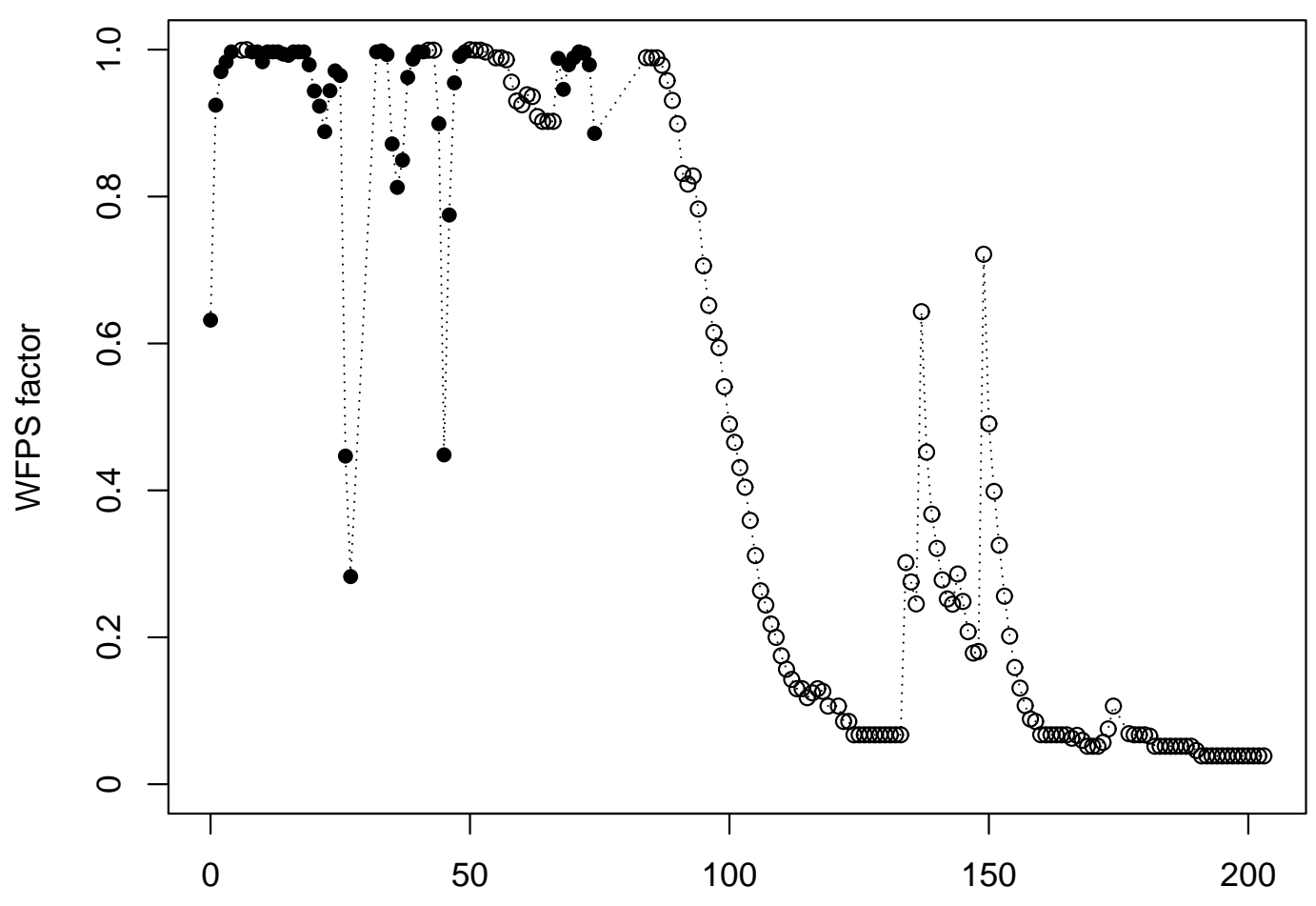

Days post 1st January 2013

Figure 4: 